\title{
Driven Production of Cold Antihydrogen and the First Measured Distribution of Antihydrogen States
}

\author{
G. Gabrielse, ${ }^{1, *}$ N. S. Bowden, ${ }^{1}$ P. Oxley, ${ }^{1}$ A. Speck, ${ }^{1}$ C. H. Storry, ${ }^{1}$ J. N. Tan, ${ }^{1}$ M. Wessels,,${ }^{1}$ D. Grzonka, ${ }^{2}$ W. Oelert, ${ }^{2}$ \\ G. Schepers, ${ }^{2}$ T. Sefzick, ${ }^{2}$ J. Walz, ${ }^{3}$ H. Pittner, ${ }^{4}$ T.W. Hänsch,,${ }^{4,5}$ and E. A. Hessels ${ }^{6}$ \\ (ATRAP Collaboration) \\ ${ }^{1}$ Department of Physics, Harvard University, Cambridge, Massachusetts 02138 \\ ${ }^{2} I K P$, Forschungszentrum Jülich GmbH, 52425 Jülich, Germany \\ ${ }^{3}$ CERN, 1211 Genève 23, Switzerland \\ ${ }^{4}$ Max-Planck-Institut für Quantenoptik, Hans-Kopfermann-Strasse 1, 85748 Garching, Germany \\ ${ }^{5}$ Ludwig-Maximilians-Universität München, Schellingstrasse 4/III, 80799 München, Germany \\ ${ }^{6}$ York University, Department of Physics and Astronomy, Toronto, Ontario M3J 1P3, Canada
}

(Received 25 October 2002; published 19 November 2002)

\begin{abstract}
Cold antihydrogen is produced when antiprotons are repeatedly driven into collisions with cold positrons within a nested Penning trap. Efficient antihydrogen production takes place during many cycles of positron cooling of antiprotons. A first measurement of a distribution of antihydrogen states is made using a preionizing electric field between separated production and detection regions. Surviving antihydrogen is stripped in an ionization well that captures and stores the freed antiproton for background-free detection.
\end{abstract}

DOI: $10.1103 /$ PhysRevLett.89.233401

PACS numbers: $36.10 .-\mathrm{k}$

Observations of cold antihydrogen $(\overline{\mathrm{H}})$ were recently reported by the ATHENA [1] and ATRAP [2] collaborations. Both used nested Penning traps, proposed [3] and developed $[4,5]$ to allow oppositely charged antiprotons $(\bar{p})$ and positrons $\left(e^{+}\right)$to interact while confined. Both observed $\overline{\mathrm{H}}$ production during the positron cooling of antiprotons in a nested Penning trap, following the earlier ATRAP demonstration [6]. The two experiments differed sharply in the way that cold $\overline{\mathrm{H}}$ was detected. ATHENA identified $\bar{p}$ and $e^{+}$annihilations within $\pm 8 \mathrm{~mm}$ and $5 \mu \mathrm{s}$ as $\overline{\mathrm{H}}$, subtracting a background (from $\bar{p}$ annihilations generating $e^{+} e^{-}$) that was larger than the signal. No information about the $\overline{\mathrm{H}}$ states was provided [1]. ATRAP used a background-free, field-ionization method to detect more $\overline{\mathrm{H}}$ in an hour than all other reported $\overline{\mathrm{H}}$ observations. The first glimpse of $\overline{\mathrm{H}}$ states was provided insofar as states ionized by electric fields between 35 and $95 \mathrm{~V} / \mathrm{cm}$ were detected [2].

More knowledge of $\overline{\mathrm{H}}$ excited state distributions is required to prepare states that can be trapped and used for precision spectroscopy. This long term goal [7] remains attractive for greatly improved $C P T$ tests with baryons and leptons [8] and sensitive tests of extensions to the standard model [9], building on accurate hydrogen spectroscopy [10]. It may even be possible to directly observe the gravitational force on cold antimatter atoms [11].

In this Letter, a measured distribution of $\overline{\mathrm{H}}$ states is reported for the first time, for $\overline{\mathrm{H}}$ produced at a high rate by driving $\bar{p}$ into collisions with cold $e^{+}$. The $\overline{\mathrm{H}}$ states are analyzed as they pass through an electric field that is varied without changing the separated $\overline{\mathrm{H}}$ production and detection. The $\bar{p}$ are resonantly driven through trapped $e^{+}$, back and forth from one side of a nested Penning trap to the other, in a new and efficient $\overline{\mathrm{H}}$ production method. $\overline{\mathrm{H}}$ forms during the positron cooling of antiprotons over many cycles, until most of the trapped $\bar{p}$ have formed $\overline{\mathrm{H}}$ or are otherwise lost from the trap. A higher $\overline{\mathrm{H}}$ production rate, per $\bar{p}$ coming to our apparatus, compensates for the reduced detection solid angle caused by the clean spatial separation of production and detection. The high rate and observed Rydberg states are what is expected for a three-body recombination mechanism $[3,12,13]$.

The apparatus and many techniques are similar to those ATRAP used to first demonstrate positron cooling of antiprotons in a nested Penning trap [6], and to observe the cold $\overline{\mathrm{H}}$ produced during this cooling [2]. A $B=5.4 \mathrm{~T}$ magnetic field from a superconducting solenoid is directed along the symmetry axis of a stack of gold-plated copper rings (Fig. 1). Applied voltages form Penning traps [Figs. 2(a) and 2(b)] that confine the $\bar{p}, e^{-}$, and $e^{+}$, and control their interactions. The electrodes and surrounding vacuum enclosure are cooled to $4.2 \mathrm{~K}$ via thermal contact to liquid helium. Cryopumping reduces the pressure within the trap to less than $5 \times 10^{-17}$ Torr, as measured in a similar apparatus using the lifetime of trapped $\bar{p}$ as a gauge [14].

Antiprotons from CERN's Antiproton Decelerator (AD) are slowed, trapped, electron cooled, and stacked $[8,15]$ in the volume below the rotatable electrode. Above, positrons from a ${ }^{22} \mathrm{Na}$ source slow and form Rydberg 


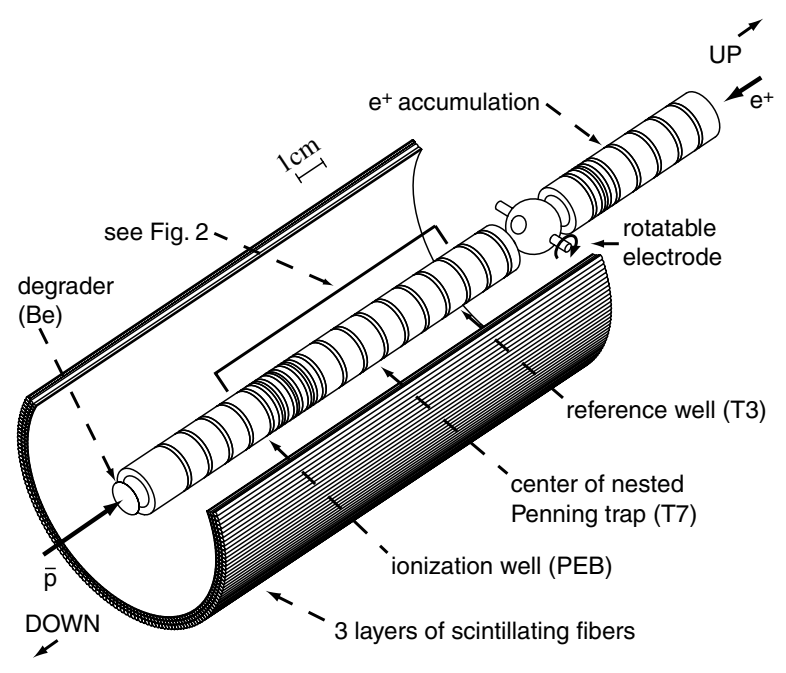

FIG. 1. Antiprotons are loaded from below (left), into the trap electrodes below the rotatable electrode. Positrons are simultaneously loaded from above (right) into the electrodes above the rotatable electrode. $\overline{\mathrm{H}}$ formation is observed within the region detailed in Fig. 2.

positronium atoms that are then ionized to accumulate $e^{+}$ [16], at the same time as the $\bar{p}$ accumulate.

The nested Penning trap [3-6] is central to $\overline{\mathrm{H}}$ production [Figs. 2(a) and 2(b)], as it was for the two earlier experiments [1,2]. The $e^{+}$are in an inverted well at the center of a larger well for $\bar{p}$, to allow $e^{+}$and $\bar{p}$ to be confined and interact despite their opposite charge signs. For these studies, typically 300000 cold $e^{+}$are located in the center well (within electrode T7). Typically $200000 \bar{p}$ are either divided between the two sides of the nested Penning trap (within T6 and T8) or placed in one side well.

The ionization and normalization wells [Figs. 2(a)2(c)], to the right and left of the nested Penning trap,

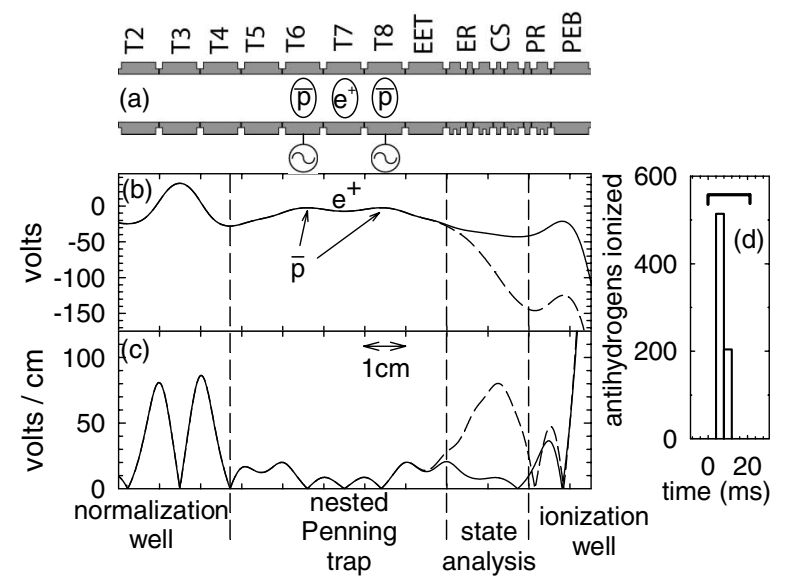

FIG. 2. Trap electrodes (a). Two values of the potential (b) and electric field magnitude (c) on axis. In a one-hour trial, 718 $\bar{p}$ from $\overline{\mathrm{H}}$ are captured in the ionization well (d). are carefully constructed to prevent $\bar{p}$ not bound in $\overline{\mathrm{H}}$ from being captured. A $\bar{p}$ heated out of the nested Penning trap will escape over the normalization well, unless there is a mechanism to lower the $\bar{p}$ energy within this well. To make capture harder the potential on the left of this well is lower by $3 \mathrm{~V}$ (on axis) than that on its right side. Getting a $\bar{p}$ into the ionization well not only requires an energy loss within the well, but also requires that the $\bar{p}$ climb a substantial potential barrier. Positron cooling keeps the $\bar{p}$ from being heated and thus makes it less likely that $\bar{p}$ will be able to pass through the ionization and normalization wells when $e^{+}$are in the nested well.

Electric fields within the ionization and normalization wells can ionize $\overline{\mathrm{H}}$ passing through, leaving freed $\bar{p}$ in one of these wells. Figure 2(c) shows the electric field on the trap axis; in the critical state-analysis region, it varies by only about $10 \%$ off the axis. Numerical modeling of $\overline{\mathrm{H}}$ trajectories shows that $\bar{p}$ in the ionization well come from $\overline{\mathrm{H}}$ stripped by fields between 25 and $150 \mathrm{~V} / \mathrm{cm}$, while $\bar{p}$ in the normalization well come from $\overline{\mathrm{H}}$ stripped by fields between 35 and $140 \mathrm{~V} / \mathrm{cm}$.

$\overline{\mathrm{H}}$ state analysis, a central feature of this work, is done by varying the potential offset between the nested well and the ionization well. This varies the state-analyzing field that $\overline{\mathrm{H}}$ encounter on their way to the ionization well, as illustrated by two examples in Fig. 2(c). Any $\overline{\mathrm{H}}$ stripped by this field is unable to deposit its $\bar{p}$ in the ionization well, causing the measured number $N$ of $\bar{p}$ in this well to decrease. (The stripping field in this well is stronger than are the state-analysis fields.) The number $N_{\text {norm }}$ of $\bar{p}$ from $\overline{\mathrm{H}}$ ionization in the normalization well provides a normalization.

Crucial radiofrequency drive potentials applied alternatively to electrodes T6 or T8 [Fig. 2(a)] drive $\bar{p}$ between the sides of the nested Penning trap. During each cycle, positron cooling allows the $\bar{p}$ to settle into the opposite, undriven side well of the nested Penning trap, and some form $\overline{\mathrm{H}}$ during this cooling. Because the $\bar{p}$ are not exactly positioned at the center of these electrodes, their symmetry does not prevent driving $\bar{p}$ axial motion.

The $825 \mathrm{kHz}$ frequency of a $1 \mathrm{~V}$ peak-to-peak drive is chosen to resonate with the calculated axial bounce frequency (Fig. 3) for $\bar{p}$ oscillating along the magnetic field direction near the axis and near the bottom of either side of the nested well. The axial bounce frequency depends on $\bar{p}$ energy, here referenced to the potential energy of a $\bar{p}$ at the center of the nested Penning trap. This frequency discontinuously halves as the $\bar{p}$ are excited out of a side well into the wider region of the nested Penning trap. The $\bar{p}$ interact with the $e^{+}$when the $\bar{p}$ energy is between zero and $-0.2 \mathrm{eV}$, the latter due to the slightly negative space charge energy of the $e^{+}$. Some optimization of the drive frequency and amplitude was done, but most of a large parameter space remains to be explored. (Another option we have used, though not for this data sample, is noise broadening the drive's frequency spectrum.) 


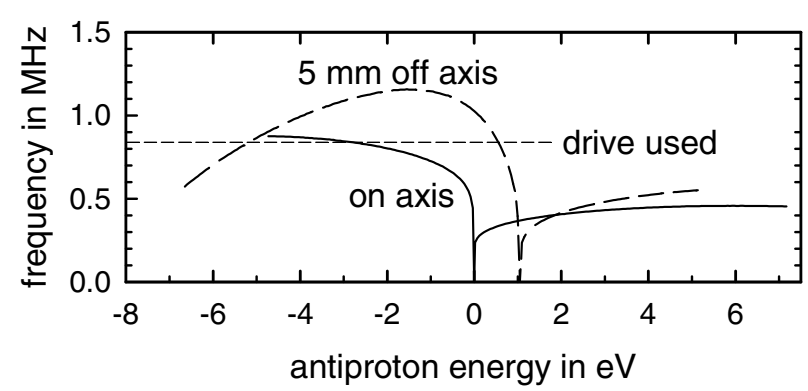

FIG. 3. Axial bounce frequency for $\bar{p}$ oscillating along the magnetic field direction in the nested Penning trap depends upon their energy, calculated with respect to their potential energy on axis at the center of the nested well. The chosen drive frequency is indicated.

We alternately drive $\bar{p}$ in one side then the other of the nested well for $10 \mathrm{~s}$, with $5 \mathrm{~s}$ between, up to 25 times. Figure 4 shows what our detectors indicate is radial $\bar{p}$ loss from the trap. Typically we transfer most $\bar{p}$ from one side to the other, though asymmetries make it common for a constant remnant of a few ten thousands of $\bar{p}$ to remain in one side well during the whole sequence. The drive cycle timing was not optimized.

To detect $\bar{p}$ deposited in the ionization and normalization wells from $\overline{\mathrm{H}}$ ionization, we ramp down these potential wells in $20 \mathrm{~ms}$, after the driving and associated particle loss are over. Ejected $\bar{p}$ annihilate upon striking electrodes, generating pions and other charged particles that produce light pulses in surrounding scintillators. The ramp is fast enough that the $1.2 \mathrm{~s}^{-1}$ cosmic ray background contributes a count in our window only 1 time in 50 - essentially no background at all. Our experimentally calibrated detection efficiency [17] corresponds to 1 in 2.7 of the stored $\bar{p}$ producing a coincidence signal in surrounding scintillators. Figure 2(d) represents $718 \bar{p}$ captured in an ionization well from $\overline{\mathrm{H}}$ ionization in a single, one-hour trial. Without $e^{+}$in the nested well, no $\bar{p}$ from $\overline{\mathrm{H}}$ ionization are detected in the ionization well.

The observed $\overline{\mathrm{H}}$ production rate, per $\bar{p}$ and per detection solid angle, is up to a factor of 12 greater than that observed using one-time positron cooling of antiprotons [2]. The $\overline{\mathrm{H}}$ rate seems very sensitive to the number of $e^{+}$in the nested well, unlike what was observed for the onetime cooling. This makes some sense insofar as the driving process continually heats the $\bar{p}$ and hence the $e^{+}$they collide with. More $e^{+}$would transfer this heat more

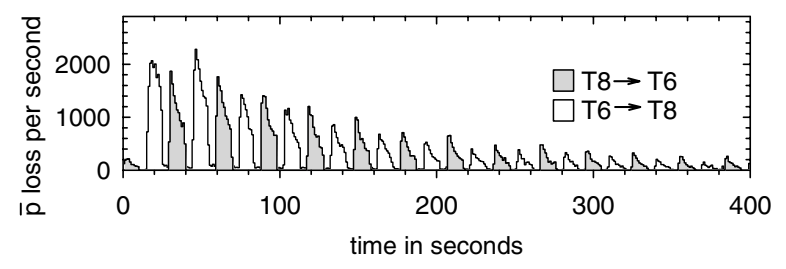

FIG. 4. Antiprotons lost while being driven from one side of the nested Penning trap to the other. rapidly into synchrotron radiation, and increase $\bar{p}$ and $e^{+}$overlap. Here much parameter space also remains to be explored. We presume that the $\overline{\mathrm{H}}$ are cold, insofar as the $\overline{\mathrm{H}}$ is likely made after very effective positron cooling of $\bar{p}$, but this must also be checked.

The first measured distribution of $\overline{\mathrm{H}}$ states is displayed in Fig. 5(a). The ratio $(R)$, of the number of $\bar{p}$ from $\overline{\mathrm{H}}$ stripped in the ionization well $(N)$ to the corresponding number in the normalization well $\left(N_{\text {norm }}\right)$, is plotted as a function of the state-analysis field $(F)$. The number of $\overline{\mathrm{H}}$ that survive this field decreases linearly until consistent with zero. The error bars prevent seeing curvature near this point, so we use a simple linear dependence going to zero to explore principal features. Thus $d R / d F$ [Fig. 5(b)] is constant up to a cutoff. As many $\overline{\mathrm{H}}$ states are ionized by fields between 30 and $35 \mathrm{~V} / \mathrm{cm}$ as between 55 and $60 \mathrm{~V} / \mathrm{cm}$, for example. No observed $\overline{\mathrm{H}}$ states require a stripping field greater than $62 \mathrm{~V} / \mathrm{cm}$.

It would be more satisfying to characterize the distribution of $\overline{\mathrm{H}}$ excited states by their principle quantum number $n$, rather than by the electric field that strips them. The first difficulty is that $n$ is not a good quantum number in the strong $B=5.4 \mathrm{~T}$ field, though we still use $n$ as a rough parametrization of binding energy, using $E=-13.6 \mathrm{eV} / n^{2}$. Ionization likely takes place in the direction of $B$ [18], giving some hope that it may not be strongly modified by $B$, but this must be investigated.

The second difficulty is that the type of Rydberg states formed determines the electric field that will ionize them, even in the absence of any magnetic field [19-21]. The field that strips a Rydberg atom entering it with principal quantum number $n$ is given (in atomic units) by
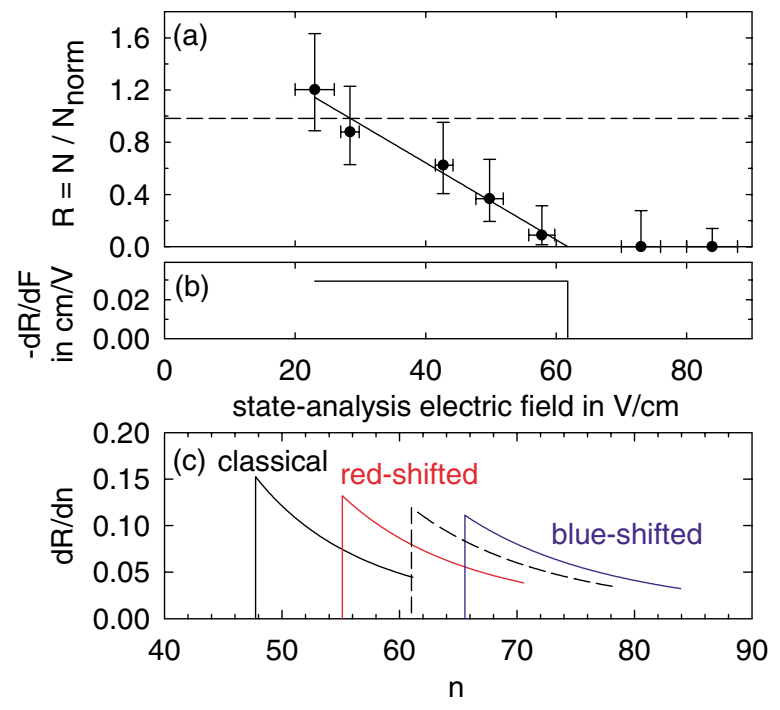

FIG. 5 (color online). (a) The ratio of ionized $\overline{\mathrm{H}}$ in ionization and normalization wells decreases linearly with state-analysis electric field $F$. (b) Distribution $d R / d F$ is flat up to a cutoff. (c) The distribution $d R / d n \sim n^{-5}$ depends upon the choice of $A$ used in Eq. (1) to relate $F$ and $n$. 


$$
F=\frac{A}{16 n^{4}} \text {. }
$$

This form and the flat distribution of Fig. 5(b) indicate that the shape of the $n$ distribution goes as $d R / d n \sim n^{-5}$.

Several examples of $d R / d n$ are shown in Fig. 5(c) since the appropriate $A$ for three-body recombination in a strong $B$ field is not known. The classical "saddle point" formula, used to give some interpretation of the $\overline{\mathrm{H}}$ produced in our one-time positron cooling of antiprotons [2], has $A=1$ and gives the lowest $n$ distribution with $n \geq 48$. Some calculations $[19,21]$ and hydrogen measurements [20] (all unfortunately for $B=0$ ) give $A$ values ranging between red and blue Stark-shifted values of $A \approx 1.8$ and 3.6, with a weak $n$ dependence in some cases [19]. This latter value is also close to that for circular Rydberg states in parallel electric and magnetic fields [18]. The dashed distribution midway between the extremes with $A \approx 2.7$ gives $n \geq 65$, and the range of possibilities suggests that these $n$ values are uncertain by at least $\pm 10 \%$. Calculations of the $\overline{\mathrm{H}}$ states produced in three-body recombination, and their ionization, are clearly needed to complete the interpretation of the measured distribution of $\overline{\mathrm{H}}$ states.

Finally, further enhancements of $\overline{\mathrm{H}}$ production seem likely with optimizations and variations on our method of arranging for many cycles of positron cooling of antiprotons. One variation would be to simultaneously drive $\bar{p}$ on both sides of the nested Penning trap. Another would be to lift $\bar{p}$ from the bottom of the nested well in a potential "bucket" for launching back into the nested Penning trap.

In conclusion, the observed $\overline{\mathrm{H}}$ production per $\bar{p}$ injected into the ATRAP apparatus is encouragingly high when $\bar{p}$ are driven into collisions with cold $e^{+}$in a nested Penning trap. The distribution of $\overline{\mathrm{H}}$ states has been measured for the first time with an analyzing electric field in a separate region between where the $\overline{\mathrm{H}}$ are produced and detected. The observed distribution $d R / d F$ is constant as a function of the state-analysis field, up to cutoff, and implications for the distribution in principal quantum number are explored. The Rydberg states and high production rate are consistent with a three-body recombination mechanism $[3,12,13]$.

The high $\overline{\mathrm{H}}$ production rate suggests the possibility to devise a way to deexcite Rydberg atoms with a range of binding energies and still get enough atoms for trapping and spectroscopy. Some temporary confinement of these highly polarizable states may be possible, but conventional trapping awaits deexcitation to the ground state, whereupon a goal is to superimpose a magnetic trap for $\overline{\mathrm{H}}$ with the Penning traps needed for its $\bar{p}$ and $e^{+}$ingredients [22].

We are grateful to CERN, its PS Division, and the AD team for delivering the $5.3 \mathrm{MeV}$ antiprotons. This work was supported by the NSF, AFOSR, the ONR of the U.S., the BMBF, MPG, and FZ-J of Germany, and the NSERC, $\mathrm{CRC}$, and PREA of Canada.

*Corresponding author.

Email address: gabrielse@physics.harvard.edu

[1] M. Amoretti et al., Nature (London) 419, 456 (2002).

[2] G. Gabrielse, N.S. Bowden, P. Oxley, A. Speck, C. H. Storry, J. N. Tan, M. Wessels, D. Grzonka, W. Oelert, G. Schepers, T. Sefzick, J. Walz, H. Pittner, T.W. Hänsch, and E. A. Hessels, Phys. Rev. Lett. 89, 213401 (2002).

[3] G. Gabrielse, S. L. Rolston, L. Haarsma, and W. Kells, Phys. Lett. A 129, 38 (1988).

[4] D. S. Hall and G. Gabrielse, Phys. Rev. Lett. 77, 1962 (1996).

[5] G. Gabrielse, D. S. Hall, T. Roach, P. Yesley, A. Khabbaz, J. Estrada, C. Heimann, and H. Kalinowsky, Phys. Lett. B 455, 311 (1999).

[6] G. Gabrielse, J. Estrada, J. N. Tan, P. Yesley, N. S. Bowden, P. Oxley, T. Roach, C. H. Storry, M. Wessels, J. Tan, D. Grzonka, W. Oelert, G. Schepers, T. Sefsick, W. Breunlich, M. Carngelli, H. Fuhrmann, R. King, R. Ursin, H. Zmeskal, H. Kalinowsky, C. Wesdorp, J. Walz, K. S. E. Eikema, and T.W. Hänsch, Phys. Lett. B 507, 1 (2001).

[7] G. Gabrielse, in Fundamental Symmetries, edited by P. Bloch, P. Paulopoulos, and R. Klapisch (Plenum, New York, 1987), p. 59.

[8] G. Gabrielse, Adv. At. Mol. Opt. Phys. 45, 1 (2001).

[9] R. Bluhm, V. A. Kostelecký, and N. Russell, Phys. Rev. D 57, 3932 (1998).

[10] M. Niering, R. Holzwarth, J. Reichert, P. Pokasov, Th. Udem, M. Weitz, T.W. Hänsch, P. Lemonde, G. Santarelli, M. Abgrall, P. Laurent, C. Salomon, and A. Clairon, Phys. Rev. Lett. 84, 5496 (2000).

[11] G. Gabrielse, Hyperfine Interact. 44, 349 (1988).

[12] M. Glinsky and T. O’Neil, Phys. Fluids B 3, 1279 (1991).

[13] P. O. Fedichev, Phys. Lett. A 226, 289 (1997).

[14] G. Gabrielse, X. Fei, L. A. Orozco, R. L. Tjoelker, J. Haas, H. Kalinowsky, T. A. Trainor, and W. Kells, Phys. Rev. Lett. 63, 1360 (1989).

[15] G. Gabrielse, N. S. Bowden, P. Oxley, A. Speck, C. H. Storry, J. N. Tan, M. Wessels, D. Grzonka, W. Oelert, G. Schepers, T. Sefzick, J. Walz, H. Pittner, T.W. Hänsch, and E. A. Hessels, Phys. Lett. B 548, 140 (2002).

[16] J. Estrada, T. Roach, J. N. Tan, P. Yesley, and G. Gabrielse, Phys. Rev. Lett. 84, 859 (2000).

[17] X. Fei, Ph.D. thesis, Harvard University, 1990.

[18] W. Ihra, F. Mota-Furtado, and P. F. O'Mahony, Phys. Rev. A 58, 3884 (1998).

[19] D. Banks and J. G. Leopold, J. Phys. B 11, L5 (1978).

[20] P. M. Koch and K. A. H. van Leeuwen, Phys. Rep. 255, 289 (1995).

[21] T.F. Gallagher, Rydberg Atoms (Cambridge University Press, New York, 1994).

[22] T. M. Squires, P. Yesley, and G. Gabrielse, Phys. Rev. Lett. 86, 5266 (2001). 